\title{
REVIEW
}

\section{The role of small airways in obstructive airway diseases}

\author{
P-R. Burgel
}

ABSTRACT: This review article is a summary of a seminar organised by the European Respiratory Society on "The role of small airways in obstructive airway diseases" which was held in October 2010 in Amsterdam, the Netherlands.

The aims of the seminar were to identify important questions related to small airways involvement in asthma and chronic obstructive pulmonary disease (COPD), and to discuss future approaches based on current and evolving knowledge. Data obtained by pathological and physiological measurements in small airways and their relevance to clinical manifestations and therapeutics in asthma and COPD were reviewed.

It was concluded that our knowledge on the roles of small airways in asthma and COPD is limited. Studies of large numbers of well-characterised subjects using multiple methods (genetic characterisation, cell biology and physiology, imaging) and integration of the data using mathematical models are suggested to be of interest. The availability of these techniques coupled with our ability to better target inhaled molecules to small airways provide a unique opportunity for a reappraisal of the relevance of small airways in chronic airway diseases.

KEYWORDS: Airway inflammation, airway remodelling, alveoli, asthma, bronchioles, chronic obstructive pulmonary disease

$\mathrm{T}$ he present review is a summary of a seminar organised by the European Respiratory Society on "The role of small airways in obstructive airway diseases" held in October 2010 in Amsterdam, the Netherlands. The Chairs of the seminar were E.H. Bel and P.J. Sterk, and the seminar participants are listed in the Acknowledgements. The aims of the seminar were to identify important questions related to small airways involvement in asthma and chronic obstructive pulmonary disease (COPD), and to discuss future approaches based on current and evolving knowledge. This article contains the results of those discussions.

Small airways are usually defined as airways $<2 \mathrm{~mm}$ in internal diameter without cartilage [1]. Although small airways contribute little to airway resistance in healthy subjects, studies using invasive measurement of airway resistance have shown that small airways are the major site of airflow limitation in both asthma and COPD [2]. Despite their importance, small airways have proven difficult to study [3]. Herein, I will examine current knowledge obtained by pathological and physiological measurements, and their relevance to clinical manifestations and therapeutics in asthma and COPD. Finally, the recent work by a pioneer of small airways disease, J. Hogg, who proposes an integrative approach of small airways disease in COPD, will be discussed.

\section{PATHOLOGY OF SMALL AIRWAYS IN ASTHMA AND COPD}

In healthy subjects, small conducting airways have a thin wall and offer little resistance to airflow that is laminar (and not turbulent). Importantly, resistance to airflow varies inversely with the fourth power of the airway radius. A decrease in airway radius may be the result of increased amount of mucus and inflammatory cells obstructing the airway lumen, increased thickness of the submucosa related to inflammation, fibrosis or increase in smooth muscle mass, and distortion and narrowing of the lumen by the loss of alveolar attachments.

Most of the pathological studies performed in asthmatic subjects were performed using autopsy tissue collected in patients with acute fatal asthma. These studies have shown extensive

\section{AFFILIATIONS}

Service de Pneumologie, Hôpital Cochin, AP-HP, and

Université Paris Descartes, Paris, France.

CORRESPONDENCE

P-R Burgel

Service de Pneumologie

Hôpital Cochin

27 rue du Faubourg St Jacques 75014 Paris

France

E-mail:

pierre-regis.burgel@cch.aphp.fr

Received:

Nov 192010

Accepted after revision:

Dec 192010

PROVENANCE

Publication of this peer-reviewed article was supported by Chiesi Farmaceutici SpA, Italy (principal sponsor, European Respiratory Review issue 119).

This version of this article replaces that originally posted online on March 1 , 2011. Some of the dosing information presented in the original version was incorrect (pages 28 and 29). This error has now been corrected. In addition, the authors have also made minor editorial changes for this latest version. 
luminal plugging by muco-inflammatory exudates and goblet cell hyperplasia in airway epithelium in both large and small airways [4-6]. Airway wall thickening with increase in smooth muscle mass and inflammatory cell infiltration by $\mathrm{T}$ lymphocytes and eosinophils were also present in large and small airways [7]. Post mortem studies have shown that the outer wall of the small airways was a major site of inflammation (especially with eosinophils) [8]. Inflammation in fatal asthma extended to the adjacent alveoli [9] and to the perivascular areas [10]. Studies have also shown loss of alveolar attachments in fatal asthma [11], which positively correlated with both mast cell and eosinophilic inflammation, suggesting roles for these inflammatory cells in alveolar disruption. Loss of alveolar attachment could result in decreased elastic recoil and premature airway closure [11]. Furthermore, alterations of the extracellular matrix (e.g. decreased decorin expression in airway wall) have been described in small airways in fatal asthma [12], possibly contributing to airway fibrosis via transforming growth factor- $\beta$ up-regulation. It has also been suggested that changes in matrix molecules within the airway smooth muscle may result in increased bronchoconstriction [12], exaggerating the effect of muco-inflammatory exudates on bronchial obstruction. The role of surfactant in fatal asthma has not been carefully studied, but may prove important because loss of surfactant may eventually contribute to small airways closure. Importantly, most patients who died of fatal asthma and who were included in pathological studies had not received any therapies, implicating that the effects of treatments on small airway inflammation and remodelling has not been established. Furthermore, it is likely that the causes of death in asthmatics have evolved due to therapeutic changes and that pathological changes may be different in subjects with different causes of exacerbation.

An important point is that data obtained in patients with fatal asthma, which probably represented acute exacerbations of poorly controlled asthma, cannot be extrapolated to patients with chronic, severe asthma. Unfortunately, very little data exist on small airways pathology in living asthmatics. Data obtained in surgical specimens of asthmatic subjects undergoing lung resection indicated increased eosinophils and Thelper (Th)2 cells polarised-CD4+ T lymphocytes in small airways versus large airways [13-15]. A limited number of studies were performed using transbronchial biopsies in selected asthmatic subjects [16, 17]. In patients with nocturnal (poorly controlled) asthma, the peribronchiolar alveoli were infiltrated with eosinophils and CD4+ T-cells [17]. In these patients, nocturnal drop in forced expiratory volume in $1 \mathrm{~s}$ (FEV1) best correlated with CD4+ T-cells in alveoli, but not with CD4+ T-cells in large bronchi [17]. Although it is clear that inflammation and remodelling are increased in small airways in acute fatal asthma and in severe and poorly controlled asthma, no pathological data exist on small airways in milder and/or in well-controlled asthmatics. Importantly, it remains unknown whether all subjects with asthma have small airways involvement or whether a "small airways phenotype" exists.

Studies of small airways in COPD subjects have long been performed using autopsy specimens and lung tissue removed at surgery for cancer therapy. Pathological hallmarks of COPD include small airways abnormalities and destruction of lung parenchyma (emphysema), which would contribute to airflow limitation via distinct mechanisms. The parenchymal component of airflow limitation is due to loss of alveolar attachment of bronchi, resulting in destabilisation of the airway and premature closing. Studies have found that increased airway inflammation was strongly correlated with destruction of alveolar attachment, suggesting a role for leukocyte-derived proteases in alveolar disruption [18]. Two different subtypes of emphysema (panlobular and centrilobular) have been described. Panlobular emphysema is characterised by destruction of the whole pulmonary lobule, whereas centrilobular emphysema is characterised by destruction of the centre of the lobule. Interestingly, recent unpublished data suggested that the destructive lesion in centrilobular emphysema was surrounded by fibrotic tissue containing increased numbers of mast cells, suggesting a mast cell-driven repair process of the alveolar walls. These findings were not observed in panlobular emphysema. The bronchiolar component of airflow limitation is characterised by small airways wall thickening with epithelial changes (e.g. goblet cell hyperplasia and squamous cell metaplasia), obstruction of airway lumen by muco-inflammatory exudates, infiltration of airway wall by inflammatory cells, increased airway smooth muscle and peribronchiolar fibrosis [19, 20]. Airway inflammation increased with the severity of airflow limitation, and was characterised by increased infiltrating macrophages, neutrophils, B and T lymphocytes and lymphoid follicles [20]. Importantly, CD8+T lymphocytes accumulate in large and small airways, in alveoli and around blood vessels [21, 22]. These CD8+ T lymphocytes are polarised toward a Th1 profile (producing interferon- $\gamma$ ) [23] and have the potential to be cytotoxic via the secretion of perforins and granzyme, which are able to damage lung tissue [24].

Infiltration of small airways by inflammatory cells is initially related to smoking exposure in patients with cigarette smokerelated COPD, but is rather independent from the intensity of smoking and may be self-perpetuating after smoking cessation. Recently, it has been proposed that self-perpetuation of inflammation in COPD small airways was related to autoimmune mechanisms [25]. A three-step approach leading to the development of COPD has been proposed [25]. In this hypothesis, Step 1 (which is present in all smokers) is characterised by innate immune responses involving neutrophils and macrophages in response to cigarette smoke-induced tissue damage. Step 2 (which may occur in some susceptible smokers) involves a shift from innate to adaptive immunity mediated by dendritic cells; T-regulatory cells may prevent excessive immunity of T-cells and the progression towards Step 3. Step 3 occurs in a minority of smokers: control mechanisms regulating $\mathrm{T}$-cell activity within the lung may be eluded, resulting in T-cell driven (autoimmune) lung injury [25]. The hypothesis of a role of autoimmune mechanisms in emphysema has been explored in animal and human studies which are exposed in the next paragraph.

In murine models, autoimmunity directed toward endothelial cells resulted in emphysema [26]. Furthermore, adoptive transfer of T-cells from cigarette smoke-exposed mice to mice deprived of T-cells (Rag 2 -/-) resulted in emphysema without further exposure to cigarette smoke [27]. LEE et al. [28] have described anti-elastin auto-antibodies in subjects with emphysema, and immunoglobulin G auto-antibodies with avidity for 
pulmonary epithelium and the potential to mediate cytotoxicity were prevalent in patients with COPD [29]. However, two recent studies have failed to reproduce such findings [30, 31] suggesting that the subjects enrolled in previous studies belonged to specific phenotypes of COPD patients. Importantly, the presence of auto-antibodies in the serum of subjects with COPD does not imply that a causal relationship exists between autoimmunity and emphysema in humans. There is some evidence that anti-elastin antibodies may be present in patients without detectable emphysema; it was suggested that these antibodies are markers of tissue damage, rather than being directly responsible for tissue damage. Furthermore, antibody directed toward self antigens may be triggered by recognition of pathogen motives (e.g. from bacteria), a phenomenon known as "molecular mimicry". In this regard the recent data that suggested that normal and diseased airways were not sterile [32] may be of particular relevance. At this stage, it remains to be established whether autoimmunity is an epiphenomenon or an important component of the pathobiology of COPD [32]. An interesting, but unanswered, question is whether autoimmune antibodies are present in asthmatic subjects who develop chronic airflow obstruction.

The pathological changes found in patients with COPD are probably related to innate and adaptive immune responses, but are also determined by the microenvironment that modulates the type of immune response to produce destructive responses in parenchyma (emphysema) and fibrosis in small airways. Several questions remain to be answered. The pathology of small airways during COPD exacerbations is unknown because such tissue is difficult to obtain, except from autopsy specimens. An important limitation is that these specimens may show many changes related to post mortem alterations rather than exacerbation-related changes. Furthermore, pathological data on small airways in COPD were obtained in cross-sectional studies, and no longitudinal data are available. As a result, the relationship between pathological changes in small airways and subsequent development of airflow limitation remains unknown.

\section{RELEVANCE OF SMALL AIRWAYS IN ASTHMA}

The clinical relevance of small airways in asthma has proven difficult to study using currently available techniques. Assessment of peripheral lung mechanics in isolated lungs using the retrograde catheter method have demonstrated that although small airways contributed little to airflow resistance in normal lungs (so-called "silent zone"), these airways were major contributors to airflow limitation in asthma and COPD $[33,34]$. Investigators have explored the relevance of small airways in asthmatics, using less invasive techniques including measurements of uneven ventilation, air trapping, alveolar nitric oxide (NO), respiratory impedance, imaging techniques and electronic nose.

In asthmatic subjects, very few data relate pathogenesis to small airways. NO has been proposed as a marker of small airways involvement in asthma. Thus, BATTAGLIA et al. [35] measured exhaled NO and small airways function using single breath nitrogen washout in 16 subjects with mild asthma. These authors found that exhaled nitric oxide fraction was positively associated with the phase III slope $\left(\mathrm{dN}_{2}\right)$ of single breath washout curve, indicating an association between exhaled NO and small airways function [35]. However, exhaled $\mathrm{NO}$ is not selective for small versus large airways. More recently, the two-compartment model allowed separation of airway versus alveolar NO [36]. VAN VEEN et al. [37] reported data showing that alveolar $\mathrm{NO}$ was associated with air trapping (as measured by residual volume (RV)/total lung capacity (TLC) \% predicted), which is a marker of premature airway closure (see below). However, a potential relationship of alveolar NO to inflammation in distal airways is subject to caution: NO is produced by resident airway cells (e.g. epithelial and endothelial cells) and mechanical stress during airway opening and closure, which is known to trigger many processes, may affect alveolar NO.

An important question is whether small airways involvement in asthma is restricted to a particular phenotype or whether it occurs in all patients. Recently, investigators have used cluster analyses to identify specific phenotypes in asthmatic subjects [38], but markers of small airways function were not included. IN T'VEEN et al. [39] studied a group of asthmatic patients with recurrent exacerbations (more than two exacerbations in the previous year; difficult-to-control asthma, $n=13$ ) compared with a group of equally severely asthmatic controls without recurrent exacerbations (stable asthma, $n=15$ ). All subjects were studied during a clinically stable period of their disease. The authors reported that asthmatic individuals with recurrent exacerbations had increased closing volume and closing capacity (CC) as compared with equally severely asthmatic but stable controls, even after bronchodilation during wellcontrolled episodes [39]. The findings imply that airway closure at relatively high lung volumes under clinically stable conditions might be a risk factor for frequent severe exacerbations in asthmatic patients, showing an association between small airways and a severe asthma phenotype.

Until recently, small airways function has not been used to differentiate between asthma and COPD. Respiratory impedance, particularly its component represented by respiratory reactance, may reflect small airways function. MUSKULUS et al. [40] hypothesised that monitoring of airway function by respiratory impedance during continuous tidal breathing may discriminate asthma and COPD. The authors used fivedimensional reconstruction of respiratory impedance dynamics and were able to differentiate between asthma and COPD subjects [40]. Although no subjects without respiratory disease were included in this study, these data suggest that the dynamics of respiratory impedance contains valuable information for the diagnosis and monitoring of patients with asthma and COPD. Another recent study further suggested that noninvasive measurement of volatile organic compounds using electronic nose may also be helpful in discriminating asthma from COPD [41]. Whether such an exhaled molecular signature is derived from volatiles produced in the periphery of the lung remains to be established.

Limited data connecting the clinical course of asthma with small airways disease are available. BouRdiN et al. [42] showed that asthmatic subjects with a history of two or more exacerbations per year had a significant increase in the slope of phase III nitrogen washout (dN2). Because this study had a cross-sectional design, it is as yet unclear whether this 
represents merely an association or whether abnormalities in small airways may be used as a predictor of subsequent exacerbations. In addition, VAN VEEN et al. [43] had observed that exhaled NO predicts lung function decline in severe asthmatics during 5 yrs of prospective follow-up [43]. Interestingly, this relationship was only found in subjects with baseline FEV1 $>80 \%$ pred, whereas no relationship was found in subjects with $\mathrm{FEV} 1 \leqslant 80 \%$, suggesting that these findings may be related to small airways rather than larger airways [43].

\section{RELEVANCE OF SMALL AIRWAYS IN COPD}

Pathological evidence relates both small airways disease and emphysema to airflow limitation in COPD (see above) [19, 20, 44]. During the 1970's and 1980's, investigators physiologically studied small airways using single breath washout tests. However, it was difficult to obtain reproducible data and it was suggested that impairment in small airways function was detectable only when the FEV1/forced vital capacity (FVC) ratio was $<70 \%$ [45]. These limitations lead to discontinuation of single breath washout tests. As a result, only small numbers of observations are available with these tests in COPD subjects and even fewer in the modern era. However, modern questions may be asked using this old technique. For example, the relationship of small airways function to airflow limitation, as assessed by FEV1/FVC < lower limit of normal, is an area for further investigation which will require testing in large numbers of subjects.

The functional effects of small airways disease in COPD subjects have been recently studied by O'DONNELL et al. [46]. These authors studied bronchodilator response, dyspnoea and exercise capacity in 16 mild symptomatic COPD subjects (FEV1 90\% pred, FEV1/FVC 60\%, diffusing capacity of the lung for carbon monoxide (DL,CO) $100 \%$ pred, specific airway resistance $300 \%$ pred) [46]. In these patients with predominant small airways disease, bronchodilator treatment was associated with modest but consistent improvements in dynamic inspiratory capacity, tidal volume and dyspnoea intensity during exercise [46].

Assessment of emphysema and airway wall thickness using computed tomography (CT) is a promising tool for studying the distal lung compartment. Unpublished data assessing the relationship of CT-defined emphysema to lung function are of potential interest. In this study, airway wall thickness at an internal perimeter of $10 \mathrm{~mm}$ was inversely correlated to FEV1 in subjects who had minimal evidence of emphysema, but not in subjects with significant emphysema. This suggests that small conducting airways are a determinant of airflow limitation mostly when emphysema is absent, whereas loss of alveolar attachment may be more important when emphysema is present. Forced oscillation is a simple technique that may be used for measuring small airways function in COPD subjects [47]. Using forced oscillation, DellacA et al. [47] showed that bronchodilators reduced inspiration resistance at $5 \mathrm{~Hz}$, paralleled by important changes in end-expiratory lung volume in eight out of 20 flow-limited patients at baseline, indicating that factors associated with small airways disease, such as increased peripheral resistance, can be modified by bronchodilators with resulting improvement in lung volume.

It can be concluded that small airways disease is a key factor leading to airway obstruction in early COPD. Although triggered by the same exposure, the relationship to tobacco may differ between emphysema and small airways disease, and this requires further study. Many more questions remain to be examined regarding the role of small airways in COPD. How heterogeneous is the damage within the lung? How does the disease progress and does this relate to the development of emphysema? At what stage can intervention reverse things? Is it worthwhile performing measurements of small airway function for identification of subjects with early COPD? Can we target therapy more effectively in terms of drug delivery? Do all insults (cigarette smoke versus other factors known to induce COPD) produce the same structural and functional abnormalities? It is likely that techniques for assessing small airways function will help in solving some of these questions, but these techniques will need to be studied in large numbers of well-characterised subjects.

\section{PHYSIOLOGICAL MEASUREMENT OF SMALL AIRWAYS FUNCTION}

Peripheral airways resistance may be measured using invasive techniques. Measurement of upstream resistance, which requires a plethysmography and an oesophageal balloon, allows partitioning of resistance and elastic recoil components of airflow limitation at a specific lung volume. Investigators have also used the wedge bronchoscope technique to obtain direct measurement of peripheral airways resistance in a distal segment [48]. These techniques are research tools that cannot be widely applied because they are technically difficult and invasive. Furthermore, an important limitation of the wedge bronchoscope technique is that measurement obtained in a given segment may not be representative of the population of distal airways because small airways disease is heterogeneous within the lung.

The characteristics of small airways obstruction include premature airway closure and air trapping, regional heterogeneity and exaggerated volume dependence of airflow limitation. Therefore, tests that focus on these characteristics can be useful surrogates to detect and quantify small airways disease. RV is elevated in the presence of premature airway closure and air trapping. Because TLC is commonly increased in obstructive disease, the RV/TLC ratio is the best measure of elevation of RV and is also considered the first step of hyperinflation. Importantly, the RV/TLC varies with age and sex, implicating that the predicted RV/TLC and the upper limit of normal obtained from reference equations are more precise comparators than the raw ratio. For example, the upper limit of normal of RV/TLC is $\sim 135 \%$ predicted for a $20 \mathrm{yr}$-old Caucasian male; thus, air trapping may be present only if RV/ TLC is $>135 \%$ pred. SORKNESS et al. [49] have recently shown that RV/TLC (\% pred) was markedly increased in severe compared to non-severe asthmatics. The authors showed that RV/TLC inversely correlated reasonably well with FVC, indicating that in the absence of volume measurements, reduction in FVC can be considered as a marker of air trapping, but these data must be validated in COPD patients [49]. The predicted value for FVC assumes normal TLC, and thus elevated TLC may result in the FVC being reduced less than expected for a given level of increase in RV, so that FVC predicted is less sensitive as an indicator for air trapping. Qualitatively, the shape of the forced expiratory flow-volume 
curve on spirometry is instructive, in that regional heterogeneity of flow time constants, progressive increases of resistance with lung deflation, and premature airway closure (all characteristics of small airways disease) together create upper concavity of the curve relative to normal. Methods have been developed to quantify changes in flow-volume curves but these have not been widely adopted. Because of its wide availability, RV/TLC remains the most interesting marker of small airways closure in clinical practice or in large multicentre trials.

The forced expiratory flow at $25-75 \%$ of FVC (FEF25-75\%) is the spirometric variable most commonly cited as an indicator of small airways obstruction. By excluding the initial peak of expiratory flow and averaging flow rate over the mid-quartile range of $\mathrm{FVC}$, the $\mathrm{FEF} 25-75 \%$ is sensitive to the same small airways characteristics that result in the concavity of expiratory flow-volume curve. As a measure of airflow limitation, FEF25-75\% correlates highly with FEV1/FVC ratio (as \% pred) but nonlinearly, such that FEF25-75\% decreases more steeply that FEV1/FVC at levels of mild obstruction. While this could improve the relative sensitivity of $\mathrm{FEF} 25-75 \%$ to detect airflow limitation, there is also a marked measurement variability in this range, such that the lower limit of normal is $<60 \%$ pred over much of the age range. It might be interesting to examine whether this variability is related to measurement characteristics or alternatively to physiological variability. Another problem with FEF25-75\% is related to alteration of FVC that occurs with air trapping and bronchodilation, which then shifts the lung volume range at which the $\mathrm{FEF} 25-75 \%$ is computed. Data obtained in the Severe Asthma Research Program of the National Heart Lung and Blood institute showed the poor correlation of FEF25-75\% with other measures of air trapping (FVC and RV/TLC), also calling into question its ability to discriminate small airways obstruction [49].

The single breath nitrogen washout test measures nitrogen concentration following a single inhalation of pure oxygen, in order to study lung structure-function relationship. The slope of the nitrogen alveolar plateau against expired volume is calculated by computer analysis of best fit line through phase III of the expiratory volume-concentration curve. Closing volume (i.e. the volume at which small airway closure occurs) is useful to measure premature airway closure that correlates well with RV/TLC [50], and which may have slightly better sensitivity. The regional heterogeneity of airflow resistance is best quantified by multiple nitrogen breath washouts (MBW). Dutrieue et al. [51] and VERBANCK et al. [52] used MBW to derive the two variables Scond (index of conductive ventilation heterogeneity) and Sacin (index of acinar ventilation heterogeneity). In asthmatic subjects, clinical applications of MBW include evaluation of ventilation heterogeneity after airway challenge [53] and analysis of the differential response to inhaled steroids when comparing extrafine versus non-extrafine particles [54]. Recently, VERBANCK et al. [55] also studied correlation between ventilation heterogeneity and alveolar NO in 30 poorly controlled asthmatics. The persistent functional abnormality of small conductive airways in patients with stable asthma was largely independent of inflammation, as measured by exhaled NO. In the alveolar compartment, a functional correlate of alveolar NO was found in a subgroup of patients with stable asthma on moderate-to-high maintenance doses of inhaled steroids [55]. In patients with COPD, MBW have been used to show improvement of small airways function after smoking cessation [56]. To date, MBW use is limited to experienced centres, but technical improvement may allow for increased use of this interesting test.

\section{IMAGING TECHNIQUES IN THE EVALUATION OF SMALL AIRWAYS IN OBSTRUCTIVE PULMONARY DISEASES}

Imaging is a novel area allowing assessment of small airways in obstructive pulmonary diseases. Imaging techniques mostly include CT and helium-magnetic resonance imaging (He-MRI). Other techniques (e.g. single photon emission CT and positron emission tomography) have not been extensively studied and will not be discussed in this article.

High-resolution CT (HRCT) allows direct assessment of large and medium airways (diameter $>2-2.5 \mathrm{~mm}$ ), but also indirect assessment of small airways. Thus, areas of mosaic lung attenuation (on inspiratory CT) and air trapping (on expiratory CT) have been studied as markers of small airways disease in both asthma and COPD. Using a semi-quantitative approach, LAURENT et al. [57] have suggested a significant positive correlation between small airways obstruction and mosaic lung attenuation in 22 subjects with mild asthma. However, this approach was limited by the semi-quantitative approach and by the absence of standardisation of lung volumes. Thus, the authors developed quantitative assessment of lung attenuation using semi-automatic tracing and analysis of density distribution (Hounsfield units), resulting in lung attenuation curves. Furthermore, spirometric gating allowed for measurement of lung attenuation at specific lung volumes. Using these techniques, Goldin et al. [58] compared 15 patients with mild asthma and six normal subjects. There was no difference in lung attenuation frequency distribution curves at baseline, but metacholine challenge induced significant decreases in the median and lowest 10th percentile regions of the attenuation curves in asthmatics but not in normal subjects [58]. Beigelman et al. [59] compared 12 subjects with mild asthma to six normal subjects. These authors reported that lung attenuation was significantly higher in asthmatics; interestingly, air trapping scores were higher after metacholine challenge, but air trapping only partially disappeared after salbutamol inhalation [59], suggesting that small airways constriction persisted. In another study, the percentage of lung field occupied by low attenuation area at inspiration showed some correlation with FEV1/FVC, suggesting an association between asthma severity and small airways [60]. Furthermore, MitsunOBU et al. [61] reported decreased CT lung density during asthma exacerbations. These findings were at least partially reversible, and changes in mean lung density and the relative lung area with low attenuation were related to the change in FEV1 and RV [61]. TUNON-DE-LARA et al. [62] have shown that 3-month treatment with inhaled corticosteroids (ICS) resulted in decreased lung attenuation and air trapping. Reduction in air trapping, but not in lung attenuation, was more pronounced with hydrofluoroalkane (HFA)-beclomethasone dipropionate (BDP) extrafine formulation (which deposited across all sizes of airways, including small airways) than with fluticasone propionate (which mostly deposited in larger airways) [62].

Investigators reported that measurements of lung attenuation obtained at inspiration and visual score better reflected abnormal results of pulmonary function tests in patients with 
less severe COPD than measurements obtained at expiration (air trapping) [63, 64]. MATSUOKA et al. [64] further analysed the relationship between airflow limitation and airway dimensions from the third to the fifth generation of bronchi in patients with COPD by using inspiratory and expiratory HRCT. The densitometric parameter of relative volume change calculated on paired inspiratory and expiratory HRCT correlated closely with airway dysfunction in COPD regardless of the degree of emphysema [64].

There are several limitations to the use of CT scans to monitor small airways disease. First, the technique uses ionising radiations, which may be important to take into account due to potential increase in malignancy, especially in younger females. Secondly, there is a need for standardisation of measurements of lung attenuation and air trapping. Factors of variation include characteristics of CT scan tube (which should be standardised in multicentre studies to avoid bias), methods of segmentation of airways and lung volumes. Thirdly, a decrease in lung attenuation may be related to reduction in ventilation but also to reduction in lung perfusion. However, changes related to perfusion are likely to have only minor effects on lung attenuation in subjects with COPD. Despite these limitations, it is suggested that a large multicentre study comparing findings obtained by assessment of small airways using CT scans and physiological measurements before surgery, to morphometric measurements of small airways in resected lung tissue of patients with COPD (and cancer) will increase our knowledge in this field. Even in such a study it will be difficult to examine the impact of heterogeneity of findings between various areas of the lung.

Magnetic resonance imaging (MRI) has the advantage of the absence of ionising radiation. It has good spatial and temporal resolution but its use is limited by poor contrast resolution. This problem can be partly solved by using i.v. gadolinium infusion or by inhalation of ${ }^{3} \mathrm{He}$, which allows analysis of ventilation distribution, estimated nonventilated lung volume and percentage diseased lung. The PHIL study evaluated HeMRI findings versus HCRT findings in 29 normal volunteers and in 52 subjects with COPD and performed correlation with FEV1/FVC indicating airway obstruction, and DL,CO indicating alveolar destruction [65]. Based on the results of ${ }^{3} \mathrm{He}-\mathrm{MRI}$, radiologists blinded from pulmonary function tests identified almost all normal volunteers and $42 \%$ of subjects with moderate COPD, whereas HRCT data identified $69 \%$ of subjects with COPD. Furthermore, ${ }^{3} \mathrm{He}-\mathrm{MRI}$ and HRCT revealed that $23 \%$ of subjects with moderate/severe structural abnormalities had only mild ventilation defects. Quantitative measurement (apparent diffusion coefficient) obtained by MRI showed better correlation with $D \mathrm{~L}, \mathrm{CO}$ than those obtained by HRCT (mean lung density; $r=0.59$ versus 0.29 ) [65]. It was concluded that ${ }^{3} \mathrm{He}$-MRI offers additional functional information, without the use of ionising radiation, whereas HRCT provides better morphological information [65]. Further studies are necessary to evaluate the role of MRI in identifying small airways disease.

\section{THERAPEUTIC INTERVENTION IN SMALL AIRWAYS DISEASE}

Anti-inflammatory treatment with ICS, with or without longacting $\beta_{2}$-agonists (LABA), is the cornerstone of asthma management. Nevertheless, some asthmatic patients do not gain optimal asthma control even with an ICS/LABA combination [66]. Inhaled therapies delivered via dry powder inhaler (DPI) or chlorofluorocarbon (CFC)-metered dose inhaler (MDI) generate large particles with a median mass aerodynamic diameter (MMAD) between 2-4 $\mu$ m leading to deposition in large airways [67]. An HFA pressurised-MDI can deliver compounds with an MMAD that is significantly smaller than other available devices [67]. These devices are able to deliver particles to both large and small airways, resulting in a significant increase in peripheral airways drug deposition [67]. A major question is whether targeting all airways (both large and small airways) can lead to further clinical benefit. Initial clinical studies were performed with extrafine formulation of ICS. In asthmatic patients, the dose of conventional non-extrafine BDP required to achieve an improvement in lung function is 2.5 times higher than the dose of HFA-extrafine formulation required to produce the same increase in FEV1 [68]. Although these data indicate that smaller particle size formulation leads to improved lung function with smaller doses of the compound, FEV1 is not a marker of small airways disease. GOLDIN et al. [69] have used HRCT to assess the relative efficacy of equipotent doses of HFA-BDP and CFC$\mathrm{BDP}$ on regional air trapping, as an indirect measure of small airways function and on regional hyperreactivity. After 4 weeks of treatment, subjects were submitted to an equal constrictor stimulus (methacholine concentration): subjects treated with HFA-BDP $(n=10)$ showed less increase in air trapping than subjects treated with CFC-BDP $(n=9)$ on the post-treatment scans, indicating that HFA-BDP may have greater efficacy in the peripheral airways [69]. Using multiple breath nitrogen washouts, VERBANCK et al. [54] have shown that asthmatic patients with abnormal acinar airway function, when treated with dry powder steroid aerosol, showed a consistent improvement after switching to an extrafine steroid aerosol. These latter studies indicated that extrafine formulation of ICS lead to improvement in small airways abnormalities, at least in some patients with specific phenotypes. JUNIPER et al. [70] conducted an open-label, 12-month randomised controlled trial comparing equipotent doses of HFA-BDP and CFC-BDP in 473 stable asthmatic subjects previously treated with CFC-BDP. The authors measured quality of life using the Asthma Quality of Life Questionnaire (AQLQ), and reported that AQLQ scores were significantly better at the end of the study in patients treated with HFA-BDP [70]. The magnitude of the improvement was less than the minimal clinically important individual difference identified for the AQLQ [70], but the numbers of subjects reaching clinically significant improvement in AQLQ was greater in the HFA-BDP group. Lung function measured with conventional clinical indexes (peak expiratory flow, FEV1, FVC and FEF25-75\%) was not significantly different between groups [70]. More recent studies were performed using fixed combination of BDP/ formoterol extrafine combination. In two large studies $(>200$ patients in each study), PAPI and co-workers [71, 72] have shown that extrafine combination of BDP/formoterol was not inferior (as measured by morning peak expiratory flow in the last 2 weeks of a 12-week treatment) to non-extrafine combination of budesonide/formoterol [71] and fluticasone/salmeterol [72]. Treatment with extrafine BDP/formoterol leads to greater improvement in FVC compared with fluticasone/salmeterol, 
suggesting an effect on small airways [72]. In a recent pilot study involving 30 asthmatic patients, SCICHILONE et al. [73] reported that a 12-week treatment with $\mathrm{BDP} /$ formoterol extrafine combination (but not with fluticasone/salmeterol) resulted in a trend toward improvement versus baseline in CC, further suggesting an improvement in small airways function. HuCHON et al. [74] performed a 24-week study in 645 patients with moderate-to-severe asthma, comparing extrafine HFA$\mathrm{BDP} /$ formoterol in a single inhaler to a combination of BDP and formoterol administered in larger particles via separate inhalers. HFA-BDP/formoterol was as effective as CFC-BDP plus formoterol DPI and was superior to CFC-BDP alone in improving lung function (as measured by morning peak expiratory flow). For the first time with a single inhaler, HFA-BDP/formoterol was significantly superior to separate components for asthma control (secondary end-point) [74]. Both ex vivo [75] and in vivo [76] studies have shown that peripheral airways contain functional $\beta_{2}$ receptors. The study by HuCHON et al. [74] raises the question of the distribution and possible co-localisation of ICS receptors and $\beta_{2}$ receptors in asthmatic airways, leading to a possible synergistic action of drugs when delivered to the small airways as fixed combination. In a recent study, COHEN et al. [77] investigated whether small and large particle sizes of aerosolised adenosine-'5monophospate (AMP) provide similar severity of airway hyperresponsiveness in 37 mild-to-moderate asthmatics. Subjects were challenged using large particle $(9.9 \mu \mathrm{m})$ and small particle $(1.06 \mu \mathrm{m})$ AMP. In asthmatics reaching a provocative concentration of AMP causing a $20 \%$ fall in FEV1 with small- and large-particle AMP provocations, smallparticle ICS ciclesonide improved hyperresponsiveness only with small particle size AMP, and fluticasone only with large particle size [77]. Although the challenge protocol may need further refinement, it is suggested that this method of airway challenge with large versus small sized particles may be useful in the identification of specific asthma phenotypes. Current data indicate that inhaled therapies with extrafine particles are at least as effective compared to larger particles in asthmatic subjects. To date, there is no definitive answer to the question: "can we achieve any additional clinical benefit by targeting small airways in asthmatics?" It is probable that patients with specific phenotypes may benefit more from treatment with extrafine particles. Among these phenotypes, asthmatic patients with fixed airflow limitation, who show higher rates of decline in FEV1 and higher rates of exacerbations [78], would be interesting to study. Smokers with asthma, who are usually excluded from clinical trials, would also be a group of interest.

Treatment of COPD includes the use of inhaled ICS/LABA and long-acting muscarinic antagonists. These therapies have been shown to reduce symptoms, prevent exacerbations and improve health-related quality of life $[79,80]$. Fixed combinations of ICS/LABA have anti-inflammatory effects proven by reduction of CD8+ $\mathrm{T}$ lymphocytes in large airways of COPD patients [81, 82]. Recent data from the GLUCOLD study indicates that ICS therapy decreases inflammation and can attenuate decline in lung function in steroid-naïve patients with moderate-to-severe COPD [83]. However, these drugs do not seem to be effective in all phenotypes of COPD, since large studies have shown that they do not effectively alter the course of the disease in unselected COPD cohorts [84, 85]. The question then rises why ICS provide some, but limited efficacy in COPD, whereas in contrast ICS are highly effective in almost all patients with asthma. The limited effects of steroids in COPD have been ascribed to the interaction of cigarette smoke with ICS targets $[86,87]$. It is also likely that when emphysema is present, the loss of lung tissue cannot be restored by steroids. However, another explanation may be equally plausible. The drugs that have been investigated to date are large particle drugs that do not easily reach the peripheral airways. Thus, it would not be surprising that the drugs applied do not have a large effect in patients where the disease is especially located in small airways. Unpublished results of the GLUCOLD study may support this, since accelerated lung function loss was less effectively reduced in COPD patients with high hyperinflation and diffusion capacity disturbances at baseline, which are signs of small airways disease and air trapping due to loss of elastic recoil and lung tissue destruction.

Recent studies have indicated that lung deposition of extrafine particles BDP/formoterol is rather unaffected by airflow limitation and that homogeneous deposition is achieved in COPD subjects [88]. Only few studies have investigated the effects of extrafine particle ICS in COPD subjects. JOHN et al. [89] studied the effects of extrafine beclomethasone inhalation in a 12-week double blind, cross-over, placebo-controlled study involving 11 COPD subjects. The authors observed a $13 \%$ reduction in $\mathrm{RV} / \mathrm{TLC}$ (\% pred) with extrafine beclomethasone but not with placebo, whereas FEV1 was unchanged [89]. Health-related quality of life was also significantly improved by extrafine beclomethasone (6.4 point improvement in St Georges Respiratory Questionnaire) [89]. However, this study was small and no comparison with inhalation of beclomethasone in larger particles was performed. In another study, TATSIS et al. [90] studied the effects of switching to HFA-BDP in 40 patients with asthma $(n=14)$ and COPD $(n=26)$ treated with inhaled steroids. On randomisation, 20 patients (15 COPD) were switched to HFA-BDP and 20 patients (11 COPD) remained on their existing budesonide turbuhaler (DPI) or fluticasone propionate inhaler (DPI) treatment [90]. After 8 weeks, HFA-BDP demonstrated a greater improvement in spirometric values, respiratory symptoms and $\beta_{2}$-agonist use [90]. Further studies are needed to explore the possibility that targeting drug deposition to both large and small airways leads to clinically significant improvement in COPD. Currently, clinical studies are ongoing to examine the effects of extrafine BDP/formoterol in COPD subjects. Results of these studies will give important insights.

\section{INTEGRATIVE MEDICINE FOR SMALL AIRWAYS DISEASE IN COPD}

Airways $<2 \mathrm{~mm}$ in diameter, which represent $<10 \%$ of total airway resistance in normal individuals, contribute to the majority of the increase in total resistance in patients with COPD [44]. This increase in total resistance may be related to a reduction of small airways numbers and/or to the narrowing of small airways. Because the total resistance of small airways is determined by the sum of the inverse resistance of each individual small airway, a removal of $50 \%$ of small airways is predicted to double small airways resistance. Narrowing of all small airways is predicted to result in an even greater increase in total small airways resistance because resistance varies 
inversely with the fourth power of the airway radius. From these calculations, it appears to be critical to determine whether the numbers of small airways are reduced in numbers in those with COPD.

HRCT scans allow reconstruction of airways and counting of airway numbers. However, current HRCT scan resolution does not permit visualisation of airways $<2-2.5 \mathrm{~mm}$ in diameter. Thus, small airways numbers and dimensions cannot be analysed with HRCT scans. Micro-CT is rather similar in principle to $\mathrm{CT}$, but uses a micro-focused X-ray source. It has the advantage over HRCT to achieve a better resolution, allowing for direct images of both bronchiolar and alveolar structures [91]. However, with current technology, use of micro-CT is limited to ex vivo tissue samples because the level of radiation associated with micro-CT damages tissue [92]. HoGG et al. [92] used micro-CT to evaluate morphological changes in human lungs obtained during transplantation in subjects with severe COPD and in unused donor lungs that served as controls. The entire lungs were inflated near TLC and frozen with liquid nitrogen. Lungs were then sliced into 2-cm thick slices and core of tissue $1 \mathrm{~cm}$ in diameter was cut, fixed, and examined by micro-CT [92]. Morphometric analyses of these samples allowed determination of the surface area and numbers of terminal bronchioles and measurements of alveolar dimensions. Data obtained from four controls indicated that there were $44.6 \times 10^{3}$ terminal bronchioles in normal human lungs. These numbers are in the range of those obtained from four bronchial casts obtained by different investigators where the total numbers of terminal bronchioles in the airways were estimated between $28 \times 10^{3}$ and $66 \times 10^{3}$ per subject. In the four control lungs, the total cross-sectional lumen area of terminal bronchioles was measured approximately at $3,000 \mathrm{~mm}^{2}$, which is in the range of previous estimations

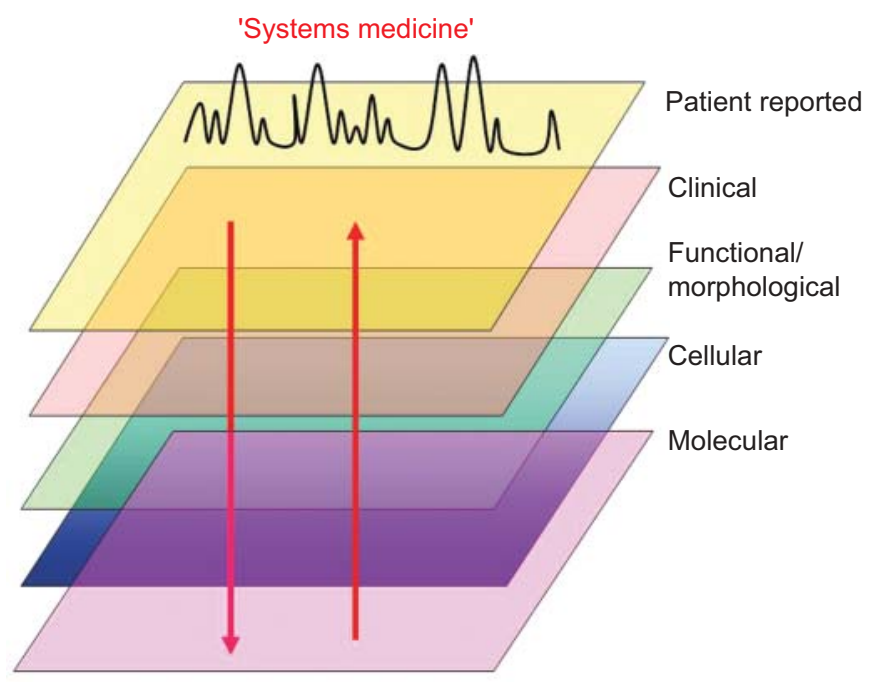

FIGURE 1. Schematic diagram of the recently described concept of "systems medicine" [93], which includes genetic characterisation, cell biology and physiology, imaging and analysis of clinical data and is a consistent, novel and interesting approach to unravel novel mechanisms in chronic respiratory disease (e.g. asthma and chronic obstructive pulmonary disease). Collection of these large amounts of data in cross-sectional and longitudinal studies and analysis using unbiased mathematical models may generate novel and unintuitive hypotheses. based on pathology. Measurement of terminal bronchiolar numbers and cross-sectional area in four subjects with severe COPD and predominant centrilobular emphysema indicated that numbers of terminal bronchioles were reduced 10-fold and cross-sectional area was reduced 100-fold [92]. Micro-CT also allowed measurement of alveolar dimension in various areas of the lungs in these subjects with various pathological phenotypes of COPD. These analyses revealed that alveolar dimensions were increased in the upper lobe, but not in the lower lobes in subjects with centrilobular emphysema. By contrast, alveolar dimension was increased throughout the lung (from the apex to the basis) in subjects with panlobular emphysema and was normal in the single subject with pure airflow obstruction. In subjects with predominant centrilobular emphysema, it was further shown that before emphysematous destruction can be appreciated with the naked eye, terminal bronchioles are reduced in number and those that remain have thickened walls. A working hypothesis is that thickening of airway walls is part of defence mechanisms against the destructive process triggered by cigarette smoke.

From these studies, HogG et al. [92] concluded that it is possible to measure a reduction in small airways numbers in Global Initiative of Obstructive Lung Disease stage IV COPD subjects. Terminal and pre-terminal bronchioles narrow and may disappear early in the course of the disease. These changes can be linked to regional differences in histology and gene expression in different pathological phenotypes of COPD. Changes in histology and gene expression are associated with the inflammatory immune cell infiltration and tissue remodelling process observed during repetitive tissue damage.

\section{FUTURE DIRECTIONS}

At the end of this symposium, it appears clear that small airways are important to the pathophysiology of chronic airway diseases, at least in some groups of subjects with asthma and with COPD. However, our understanding of small airways involvement in these diseases is incomplete. Progress in this field will probably come from studies involving large numbers of well-characterised subjects who will be studied using a combination of multiple techniques. The recently described "Systems medicine" approach that includes genetic characterisation, cell biology and physiology, studies of organ structure and function, and studies of organisms in health and disease is suggested to be of interest (fig. 1) [93]. The availability of these techniques coupled with our ability to better target inhaled molecules to small airways provide a unique opportunity for a reappraisal of the relevance of small airways in chronic airway diseases.

\section{STATEMENT OF INTEREST}

P-R. Burgel has received less than $€ 5,000$ during the past 5 years for fees for speaking by Chiesi Pharmaceuticals. Travel to the ATS congress was also funded by Chiesi Pharmaceuticals. P-R. Burgel received fees for advisory board participation or lectures from AstraZeneca, Boehringer Ingelheim, Chiesi, Novartis and Nycomed.

\section{ACKNOWLEDGEMENTS}

The meeting summarised in the present review was chaired by E.H. Bel and P.J. Sterk (Academic Medical Centre, University of Amsterdam, the Netherlands). The following people also participated in the meeting: P.M.A. Calverley (School of Clinical Sciences, University of Liverpool, 
Liverpool, UK); M. Contoli (Research Centre on Asthma and COPD, University of Ferrara, Ferrara, Italy); M. Cosio (Respiratory Division, McGill University, Montreal, Quebec, Canada); J. Hogg (Dept of Pathology and Laboratory Medicine, MacDonald Research Laboratory, St Paul's Hospital, Vancouver, British Colombia, Canada); T. Mauad (Dept of Pathology, Sao Paulo University Medical School, Sao Palo, Brazil); D.S. Postma (Dept of Pulmonary Diseases, University Medical Center Groningen, Groningen, the Netherlands); M. Saetta (Dept of Cardiac, Thoracic and Vascular Sciences, University of Padova, Padova, Italy); R.L. Sorkness (University of Wisconsin, Madison, WI, USA); J.M. Tunon de Lara (Université Victor Segalen Bordeaux 2, Bordeaux, France); S. Verbank (Universitair Ziekenhuis, Brussels, Belgium).

\section{REFERENCES}

1 Ranga V, Kleinerman J. Structure and function of small airways in health and disease. Arch Pathol Lab Med 1978; 102: 609-617.

2 Yanai M, Sekizawa K, Ohrui T, et al. Site of airway obstruction in pulmonary disease: direct measurement of intrabronchial pressure. J Appl Physiol 1992; 72: 1016-1023.

3 Burgel P-R, de Blic J, Chanez P, et al. Update on the roles of distal airways in asthma. Eur Respir Rev 2009; 18: 80-95.

4 Aikawa $\mathrm{T}$, Shimura S, Sasaki $\mathrm{H}$, et al. Marked goblet cell hyperplasia with mucus accumulation in the airways of patients who died of severe acute asthma attack. Chest 1992; 101: 916-921.

5 Dunnill MS. The pathology of asthma with special reference to changes in the bronchial mucosa. J Clin Pathol 1960; 13: 27-33.

6 Kuyper LM, Pare PD, Hogg JC, et al. Characterization of airway plugging in fatal asthma. Am J Med 2003; 115: 6-11.

7 Carroll N, Cooke C, James A. The distribution of eosinophils and lymphocytes in the large and small airways of asthmatics. Eur Respir J 1997; 10: 292-300.

8 Dolhnikoff M, da Silva LF, de Araujo BB, et al. The outer wall of small airways is a major site of remodeling in fatal asthma. J Allergy Clin Immunol 2009; 123: 1090-1097.

9 de Magalhaes Simoes S, dos Santos MA, da Silva Oliveira M, et al. Inflammatory cell mapping of the respiratory tract in fatal asthma. Clin Exp Allergy 2005; 35: 602-611.

10 Shiang C, Mauad T, Senhorini A, et al. Pulmonary periarterial inflammation in fatal asthma. Clin Exp Allergy 2009; 39: 1499-1507.

11 Mauad T, Silva LF, Santos MA, et al. Abnormal alveolar attachments with decreased elastic fiber content in distal lung in fatal asthma. Am J Respir Crit Care Med 2004; 170: 857-862.

12 de Medeiros Matsushita M, da Silva LF, dos Santos MA, et al. Airway proteoglycans are differentially altered in fatal asthma. J Pathol 2005; 207: 102-110.

13 Hamid Q, Song Y, Kotsimbos TC, et al. Inflammation of small airways in asthma. J Allergy Clin Immunol 1997; 100: 44-51.

14 Minshall EM, Hogg JC, Hamid QA. Cytokine mRNA expression in asthma is not restricted to the large airways. J Allergy Clin Immunol 1998; 101: 386-390.

15 Taha RA, Minshall EM, Miotto D, et al. Eotaxin and monocyte chemotactic protein-4 mRNA expression in small airways of asthmatic and nonasthmatic individuals. J Allergy Clin Immunol 1999; 103: 476-483.

16 Balzar S, Wenzel SE, Chu HW. Transbronchial biopsy as a tool to evaluate small airways in asthma. Eur Respir J 2002; 20: 254-259.

17 Kraft M, Martin RJ, Wilson S, et al. Lymphocyte and eosinophil influx into alveolar tissue in nocturnal asthma. Am J Respir Crit Care Med 1999; 159: 228-234.

18 Saetta M, Ghezzo H, Kim WD, et al. Loss of alveolar attachments in smokers. A morphometric correlate of lung function impairment. Am Rev Respir Dis 1985; 132: 894-900.

19 Cosio M, Ghezzo H, Hogg JC, et al. The relations between structural changes in small airways and pulmonary-function tests. N Engl J Med 1978; 298: 1277-1281.
20 Hogg JC, Chu F, Utokaparch S, et al. The nature of small-airway obstruction in chronic obstructive pulmonary disease. $N$ Engl J Med 2004; 350: 2645-2653.

21 Saetta M, Baraldo S, Corbino L, et al. CD8+ve cells in the lungs of smokers with chronic obstructive pulmonary disease. Am J Respir Crit Care Med 1999; 160: 711-717.

22 Saetta M, Di Stefano A, Turato G, et al. CD8+ T-lymphocytes in peripheral airways of smokers with chronic obstructive pulmonary disease. Am J Respir Crit Care Med 1998; 157: 822-826.

23 Saetta $M$, Mariani $M$, Panina-Bordignon $\mathrm{P}$, et al. Increased expression of the chemokine receptor CXCR3 and its ligand CXCL10 in peripheral airways of smokers with chronic obstructive pulmonary disease. Am J Respir Crit Care Med 2002; 165: 1404-1409.

24 Chrysofakis G, Tzanakis N, Kyriakoy D, et al. Perforin expression and cytotoxic activity of sputum CD8+ lymphocytes in patients with COPD. Chest 2004; 125: 71-76.

25 Cosio MG, Saetta M, Agusti A. Immunologic aspects of chronic obstructive pulmonary disease. N Engl J Med 2009; 360: 2445-2454

26 Taraseviciene-Stewart L, Scerbavicius R, Choe KH, et al. An animal model of autoimmune emphysema. Am J Respir Crit Care Med 2005; 171: 734-742.

27 Motz GT, Eppert BL, Wesselkamper SC, et al. Chronic cigarette smoke exposure generates pathogenic T cells capable of driving COPD-like disease in Rag2-/- mice. Am J Respir Crit Care Med 2010; 181: 1223-1233.

28 Lee SH, Goswami S, Grudo A, et al. Antielastin autoimmunity in tobacco smoking-induced emphysema. Nat Med 2007; 13: 567-569.

29 Feghali-Bostwick CA, Gadgil AS, Otterbein LE, et al. Autoantibodies in patients with chronic obstructive pulmonary disease. Am J Respir Crit Care Med 2008; 177: 156-163.

30 Cottin V, Fabien N, Khouatra C, et al. Anti-elastin autoantibodies are not present in combined pulmonary fibrosis and emphysema. Eur Respir J 2009; 33: 219-221.

31 Greene CM, Low TB, O'Neill SJ, et al. Anti-proline-glycine-proline or antielastin autoantibodies are not evident in chronic inflammatory lung disease. Am J Respir Crit Care Med 2010; 181: 31-35.

32 Hilty M, Burke C, Pedro H, et al. Disordered microbial communities in asthmatic airways. PLoS One 2010; 5: e8578.

33 Macklem PT, Mead J. Resistance of central and peripheral airways measured by a retrograde catheter. J Appl Physiol 1967; 22: 395-401.

34 Bates JH, Suki B. Assessment of peripheral lung mechanics. Respir Physiol Neurobiol 2008; 163: 54-63.

35 Battaglia S, den Hertog H, Timmers MC, et al. Small airways function and molecular markers in exhaled air in mild asthma. Thorax 2005; 60: 639-644.

36 Tsoukias NM, George SC. A two-compartment model of pulmonary nitric oxide exchange dynamics. J Appl Physiol 1998; 85: 653-666.

37 van Veen $\mathrm{IH}$, Sterk PJ, Schot $\mathrm{R}$, et al. Alveolar nitric oxide versus measures of peripheral airway dysfunction in severe asthma. Eur Respir J 2006; 27: 951-956.

38 Haldar P, Pavord ID, Shaw DE, et al. Cluster analysis and clinical asthma phenotypes. Am J Respir Crit Care Med 2008; 178: 218-224.

39 in 't Veen JC, Beekman AJ, Bel EH, et al. Recurrent exacerbations in severe asthma are associated with enhanced airway closure during stable episodes. Am J Respir Crit Care Med 2000; 161: 1902-1906.

40 Muskulus M, Slats AM, Sterk PJ, et al. Fluctuations and determinism of respiratory impedance in asthma and chronic obstructive pulmonary disease. J Appl Physiol 2010; 109: 1582-1591.

41 Fens N, Zwinderman $\mathrm{AH}$, van der Schee $\mathrm{MP}$, et al. Exhaled breath profiling enables discrimination of chronic obstructive pulmonary disease and asthma. Am J Respir Crit Care Med 2009; 180: 1076-1082.

42 Bourdin A, Paganin F, Prefaut C, et al. Nitrogen washout slope in poorly controlled asthma. Allergy 2006; 61: 85-89.

43 van Veen $\mathrm{IH}$, Ten Brinke A, Sterk PJ, et al. Exhaled nitric oxide predicts lung function decline in difficult-to-treat asthma. Eur Respir J 2008; 32: 344-349. 
44 Hogg JC, Macklem PT, Thurlbeck WM. Site and nature of airway obstruction in chronic obstructive lung disease. N Engl J Med 1968; 278: 1355-1360.

45 Stanescu D, Sanna A, Veriter C, et al. Identification of smokers susceptible to development of chronic airflow limitation: a 13-year follow-up. Chest 1998; 114: 416-425.

46 O'Donnell DE, Laveneziana $\mathrm{P}$, Ora J, et al. Evaluation of acute bronchodilator reversibility in patients with symptoms of GOLD stage I COPD. Thorax 2009; 64: 216-223.

47 Dellaca RL, Pompilio PP, Walker PP, et al. Effect of bronchodilation on expiratory flow limitation and resting lung mechanics in COPD. Eur Respir J 2009; 33: 1329-1337.

48 Wagner EM, Liu MC, Weinmann GG, et al. Peripheral lung resistance in normal and asthmatic subjects. Am Rev Respir Dis 1990; 141: 584-588.

49 Sorkness RL, Bleecker ER, Busse WW, et al. Lung function in adults with stable but severe asthma: air trapping and incomplete reversal of obstruction with bronchodilation. J Appl Physiol 2008; 104: 394-403.

50 McFadden ER Jr, Kiker R, Holmes B, et al. Small airway disease. An assessment of the tests of peripheral airway function. Am J Med 1974; 57: 171-182.

51 Dutrieue B, Vanholsbeeck F, Verbanck S, et al. A human acinar structure for simulation of realistic alveolar plateau slopes. J Appl Physiol 2000; 89: 1859-1867.

52 Verbanck S, Paiva M. Model simulations of gas mixing and ventilation distribution in the human lung. J Appl Physiol 1990; 69: 2269-2279.

53 Verbanck S, Schuermans D, Van Muylem A, et al. Ventilation distribution during histamine provocation. J Appl Physiol 1997; 83: 1907-1916.

54 Verbanck S, Schuermans D, Paiva M, et al. The functional benefit of anti-inflammatory aerosols in the lung periphery. J Allergy Clin Immunol 2006; 118: 340-346.

55 Verbanck S, Schuermans D, Vincken W. Inflammation and airway function in the lung periphery of patients with stable asthma. J Allergy Clin Immunol 2010; 125: 611-616.

56 Verbanck S, Schuermans D, Paiva M, et al. Small airway function improvement after smoking cessation in smokers without airway obstruction. Am J Respir Crit Care Med 2006; 174: 853-857.

57 Laurent F, Latrabe V, Raherison C, et al. Functional significance of air trapping detected in moderate asthma. Eur Radiol 2000; 10: 1404-1410.

58 Goldin JG, McNitt-Gray MF, Sorenson SM, et al. Airway hyperreactivity: assessment with helical thin-section CT. Radiology 1998; 208: 321-329.

59 Beigelman-Aubry C, Capderou A, Grenier PA, et al. Mild intermittent asthma: CT assessment of bronchial cross-sectional area and lung attenuation at controlled lung volume. Radiology 2002; 223: 181-187.

60 Ueda T, Niimi A, Matsumoto H, et al. Role of small airways in asthma: investigation using high-resolution computed tomography. J Allergy Clin Immunol 2006; 118: 1019-1025.

61 Mitsunobu F, Ashida K, Hosaki Y, et al. Decreased computed tomographic lung density during exacerbation of asthma. Eur Respir J 2003; 22: 106-112.

62 Tunon-de-Lara JM, Laurent F, Giraud V, et al. Air trapping in mild and moderate asthma: effect of inhaled corticosteroids. J Allergy Clin Immunol 2007; 119: 583-590.

63 Akira M, Toyokawa K, Inoue Y, et al. Quantitative CT in chronic obstructive pulmonary disease: inspiratory and expiratory assessment. AJR Am J Roentgenol 2009; 192: 267-272.

64 Matsuoka S, Kurihara Y, Yagihashi K, et al. Airway dimensions at inspiratory and expiratory multisection $\mathrm{CT}$ in chronic obstructive pulmonary disease: correlation with airflow limitation. Radiology 2008; 248: 1042-1049.
65 van Beek EJ, Dahmen AM, Stavngaard T, et al. Hyperpolarised $3 \mathrm{He}$ MRI versus HRCT in COPD and normal volunteers: PHIL trial. Eur Respir J 2009; 34: 1311-1321.

66 Bateman ED, Boushey HA, Bousquet J, et al. Can guideline-defined asthma control be achieved? The Gaining Optimal Asthma ControL study. Am J Respir Crit Care Med 2004; 170: 836-844.

67 Leach C, Colice GL, Luskin A. Particle size of inhaled corticosteroids: does it matter? J Allergy Clin Immunol 2009; 124: Suppl. 6, S88-S93.

68 Busse WW, Brazinsky S, Jacobson K, et al. Efficacy response of inhaled beclomethasone dipropionate in asthma is proportional to dose and is improved by formulation with a new propellant. J Allergy Clin Immunol 1999; 104: 1215-1222.

69 Goldin JG, Tashkin DP, Kleerup EC, et al. Comparative effects of hydrofluoroalkane and chlorofluorocarbon beclomethasone dipropionate inhalation on small airways: assessment with functional helical thin-section computed tomography. J Allergy Clin Immunol 1999; 104: S258-S267.

70 Juniper EF, Price DB, Stampone PA, et al. Clinically important improvements in asthma-specific quality of life, but no difference in conventional clinical indexes in patients changed from conventional beclomethasone dipropionate to approximately half the dose of extrafine beclomethasone dipropionate. Chest 2002; 121: 1824-1832.

71 Papi A, Paggiaro PL, Nicolini G, et al. Beclomethasone/formoterol versus budesonide/formoterol combination therapy in asthma. Eur Respir J 2007; 29: 682-689.

72 Papi A, Paggiaro P, Nicolini G, et al. Beclomethasone/formoterol vs fluticasone/salmeterol inhaled combination in moderate to severe asthma. Allergy 2007; 62: 1182-1188.

73 Scichilone N, Battaglia S, Soriano C, et al. Effects of extra-fine inhaled beclomethasone/formoterol on both large and small airways in asthma. Allergy 2010; 65: 897-902.

74 Huchon G, Magnussen H, Chuchalin A, et al. Lung function and asthma control with beclomethasone and formoterol in a single inhaler. Respir Med 2009; 103: 41-49.

75 Sturton RG, Trifilieff A, Nicholson AG, et al. Pharmacological characterization of indacaterol, a novel once daily inhaled 2 adrenoceptor agonist, on small airways in human and rat precision-cut lung slices. J Pharmacol Exp Ther 2008; 324: 270-275.

76 Devolder A, De Backer L, Vos W, et al. Evaluation of the acute bronchodilator effect of beclomethasone/formoterol fixed combination on central and peripheral airway dimensions in asthmatic patients. Eur Respir J 2009; 34: Suppl. 53, 518s.

77 Cohen J, Postma DS, Douma WR, et al. Particle size matters: diagnostics and treatment of small airways involvement in asthma. Eur Respir J 2010; [Epub ahead of print DOI: 10.1183/ ?09031936.00204109].

78 Contoli M, Baraldo S, Marku B, et al. Fixed airflow obstruction due to asthma or chronic obstructive pulmonary disease: 5-year follow-up. J Allergy Clin Immunol 2010; 125: 830-837.

79 Calverley PM, Anderson JA, Celli B, et al. Salmeterol and fluticasone propionate and survival in chronic obstructive pulmonary disease. N Engl J Med 2007; 356: 775-789.

80 Tashkin DP, Celli B, Senn S, et al. A 4-year trial of tiotropium in chronic obstructive pulmonary disease. $N$ Engl J Med 2008; 359: 1543-1554.

81 Barnes NC, Qiu YS, Pavord ID, et al. Antiinflammatory effects of salmeterol/fluticasone propionate in chronic obstructive lung disease. Am J Respir Crit Care Med 2006; 173: 736-743.

82 Bourbeau J, Christodoulopoulos P, Maltais F, et al. Effect of salmeterol/fluticasone propionate on airway inflammation in COPD: a randomised controlled trial. Thorax 2007; 62: 938-943.

83 Lapperre TS, Snoeck-Stroband JB, Gosman MM, et al. Effect of fluticasone with and without salmeterol on pulmonary outcomes in chronic obstructive pulmonary disease: a randomized trial. Ann Intern Med 2009; 151: 517-527. 
84 Burge PS, Calverley PM, Jones PW, et al. Randomised, double blind, placebo controlled study of fluticasone propionate in patients with moderate to severe chronic obstructive pulmonary disease: the ISOLDE trial. BMJ 2000; 320: 1297-1303.

85 Pauwels RA, Lofdahl CG, Laitinen LA, et al. Long-term treatment with inhaled budesonide in persons with mild chronic obstructive pulmonary disease who continue smoking. European Respiratory Society Study on Chronic Obstructive Pulmonary Disease. N Engl J Med 1999; 340: 1948-1953.

86 Culpitt SV, Maziak W, Loukidis S, et al. Effect of high dose inhaled steroid on cells, cytokines, and proteases in induced sputum in chronic obstructive pulmonary disease. Am J Respir Crit Care Med 1999; 160: 1635-1639.

87 Keatings VM, Jatakanon A, Worsdell YM, et al. Effects of inhaled and oral glucocorticoids on inflammatory indices in asthma and COPD. Am J Respir Crit Care Med 1997; 155: 542-548.

88 De Backer W, Devolder A, Poli G, et al. Lung deposition of BDP/ formoterol HFA pMDI in healthy volunteers, asthmatic, and COPD patients. J Aerosol Med Pulm Drug Deliv 2010; 23: 137-148.
89 John M, Bosse S, Oltmanns U, et al. Effects of inhaled HFA beclomethasone on pulmonary function and symptoms in patients with chronic obstructive pulmonary disease. Respir Med 2005; 99: 1418-1424.

90 Tatsis G, Kotsifas K, Filaditaki V, et al. Efficacy of beclomethasone dipropionate HFA 200 microg once daily in chronic obstructive pulmonary disease and bronchial asthma. J Int Med Res 2007; 35: 361-373.

91 Watz H, Breithecker A, Rau WS, et al. Micro-CT of the human lung: imaging of alveoli and virtual endoscopy of an alveolar duct in a normal lung and in a lung with centrilobular emphysema initial observations. Radiology 2005; 236: 1053-1058.

92 Hogg JC, McDonough JE, Sanchez PG, et al. Micro-computed tomography measurements of peripheral lung pathology in chronic obstructive pulmonary disease. Proc Am Thorac Soc 2009; 6: 546-549.

93 Auffray C, Adcock IM, Chung KF, et al. An integrative systems biology approach to understanding pulmonary diseases. Chest 2010; 137: 1410-1416. 